Flores-Rodríguez, J.; Ramírez-Macías, G. (2021). Empty Goal: It's Influence on the Men's Handball World Championship 2019. Revista Internacional de Medicina y Ciencias de la Actividad Física y el Deporte vol. 21 (84) pp. 591-606. Http://cdeporte.rediris.es/revista/revista83/artporteria1264.htm

DOI: https://doi.org/10.15366/rimcafd2021.83.011

\title{
ORIGINAL
}

\section{PORTERÍA VACÍA: SU INFLUENCIA EN EL CAMPEONATO MUNDIAL DE BALONMANO MASCULINO 2019}

\section{EMPTY GOAL: IT'S INFLUENCE ON THE MEN'S HANDBALL WORLD CHAMPIONSHIP 2019}

\author{
Flores-Rodríguez, J. $^{1}$ y Ramírez-Macías, G. $^{2}$ \\ ${ }^{1}$ Doctorando en Ciencias de la Actividad Física y Deporte. Grupo de Investigación HUM962. \\ Deporte y Sociedad. Universidad de Sevilla (España) josefloresrodriguez@live.com \\ 2 Profesor titular. Departamento de Educación Física y Deporte. Universidad de Sevilla \\ (España) grm@us.es
}

Código UNESCO I UNESCO code: 5899 Otras especialidades pedagógicas: Educación Física y Deporte/ Other pedagogical specialties: Physical Education and Sports

Clasificación Consejo de Europa / Council of Europe classification: 17. Rendimiento Deportivo / Others: Sports Performance)

Recibido 26 de junio de 2019 Received June 26, 2019

Aceptado 10 de octubre de 2019 Accepted October 10, 2019

\section{RESUMEN}

El objetivo de la presente investigación fue conocer el impacto que ejerce en el desarrollo del juego la nueva norma, que permite la sustitución del portero por un jugador de campo (jugando a portería vacía). Empleando un instrumento de observación ad hoc se registraron todos los ataques realizados en los partidos correspondientes a la fase final del Campeonato Mundial masculino de Balonmano celebrado en 2019. Tras un análisis descriptivo, se aplicó la técnica de coordenadas polares para conocer los patrones de conducta que emergen en una situación de 6 vs. 6 , según se utilice o no el recurso de la portería vacía. Los resultados indican que la nueva norma se utiliza primordialmente en inferioridad numérica, provocando diferentes comportamientos en las fases de ataque y repliegue defensivo. Finalmente, hay que destacar que conocer las nuevas tendencias del juego puede ayudar a optimizar los programas de aprendizaje y rendimiento deportivo.

PALABRAS CLAVE: balonmano, potería vacía, rendimiento deportivo 


\begin{abstract}
This research aims to determine the influence that the new regulation has on the development of the game. This rule allows the replacement of the goalkeeper by a field player (playing empty goal). Using an ad hoc observation instrument, all the attacks made in the matches corresponding to the final phase of the World Men's Handball Championship held in 2019 were registered. After a descriptive analysis, the polar coordinates technique was applied to specify the behaviour patterns emerging in a situation of 6 vs. 6 , depending on whether or not the resource of the empty goal is used. The results indicate that the new regulation is used primarily in numerical inferiority, causing different behaviours in the phases of attack and defensive balance. Finally, it should be noted that knowing the new trends of the game can help optimise learning programmes and sports performance.
\end{abstract}

KEY WORDS: handball, empty goal, sport performance.

\title{
1 INTRODUCCIÓN
}

La modificación de las reglas de juego condicionan decisivamente los comportamientos realizados por los equipos (Beiztegui-Casado, OliverCoronado, \& Sosa-González, 2019; Marczinka \& Gál, 2018). Recientemente, en el año 2016, la Federación Internacional de Balonmano modificó el reglamento, permitiendo sustituir al portero por un jugador de campo extra, sin que ninguno de los jugadores que actúan tenga la obligatoriedad de vestir la indumentaria distintiva de portero y posibilitando por tanto, que cualquier jugador pueda abandonar el terreno de juego para que entre el portero. La sustitución del portero por un jugador de campo no es una novedad, la norma anterior permitía esta posibilidad, pero presentaba un matiz importante respecto a la actual: existía la obligatoriedad, para el jugador de campo que entraba en sustitución del portero, de vestir con la indumentaria de portero, actuando como "portero-falso" o "portero-jugador" y siendo el único que podía cambiarse por el portero "auténtico".

En el último Campeonato del Mundo masculino, celebrado en los países de Dinamarca y Alemania en el año 2019, los entrenadores han utilizado distintas estrategias ofensivas y defensivas, orientadas a sacar el máximo rendimiento a las posibilidades que ofrece esta nueva norma. A lo largo del torneo, las selecciones han sustituido al portero por un jugador de campo en tres contextos específicos: en situaciones ofensivas con igualdad numérica, asumiendo el riego de dejar su portería vacía para obtener una superioridad numérica ofensiva respecto a la defensa; en situaciones ofensivas con inferioridad numérica, para compensar la ausencia del jugador o jugadores excluidos e incluso algunas selecciones han empleado este recurso en situaciones de superioridad numérica (para jugar el ataque con una superioridad numérica de más de un jugador). 
No son muchas las investigaciones que han estudiado la sustitución del portero por un jugador de campo. Antes de la entrada en vigor de la nueva norma, Antón (2010) realizó un repaso sobre distintas estrategias empleadas a lo largo de la historia, exponiendo y argumentando las ventajas e inconvenientes de su utilización. Recientemente, Beiztegui-Casado et al. (2019) analizaron el uso del portero-jugador en el Campeonato del Mundo femenino celebrado en Dinamarca en el año 2015, encontrando que su utilización favorece la obtención de gol en situaciones de inferioridad numérica ofensiva, a la vez que no penaliza al equipo que lo utiliza, al no existir diferencia significativa en la probabilidad de recibir gol en contraataque. Ya con la nueva norma implantada, Marczinka \& Gál (2018), estudiaron su influencia durante el Campeonato del Mundo masculino celebrado en Francia en el año 2017. En su estudio exploraron la frecuencia y la eficacia con la que los equipos utilizaron este cambio de reglas, afirmando que éstos primordialmente utilizaron las posibilidades de esta nueva regla en las situaciones de inferioridad numérica, compensado así la pérdida del jugador excluido y asumiendo el riesgo de jugar en ataque con la portería vacía.

Por lo novedoso de la regla y por la escasez de estudios que aborden su tremenda influencia en la dinámica del juego (Marczinka \& Gál, 2018), el presente trabajo tiene como objetivo obtener conocimiento científico sobre el impacto que tiene, en el desarrollo del juego, el hecho de jugar con la portería vacía. Para ello, se estudiaron los partidos correspondientes a la fase final (los dos partidos de semifinales, la final y el partido por el tercer puesto) del Campeonato del Mundo masculino celebrado en 2019. Tras un estudio descriptivo sobre su incidencia, se analizaron, mediante la aplicación de la técnica de coordenadas polares, los patrones de conducta que emergen en una de las situaciones donde la nueva regla es más utilizada: 6 vs. 6 (seis atacantes contra seis defensores) cuando el equipo que ataca está en inferioridad numérica (Marczinka \& Gál, 2018) comparándolos con los patrones de conducta que emergen, en la misma situación de 6 vs. 6, cuando ambos equipos están en igualdad numérica y no utilizan el recurso de jugar con la portería vacía.

\section{MATERIAL Y MÉTODOS}

\subsection{METODOLOGÍA OBSERVACIONAL}

Se ha planteado un diseño observacional Seguimiento/Idiográfico/Multidimensional (S/I/M) (Anguera, Blanco-Villaseñor, Hernández-Mendo \& Losada, 2011). Su justificación es la siguiente: seguimiento, ya que se analizaron varios partidos; idiográfico debido a que aunque son varios los jugadores estudiados, al formar parte de un mismo equipo (cada una de las selecciones nacionales) funcionan como unidad; y por último, multidimensional porque se estudian diversos niveles de respuesta. 


\subsection{PARTICIPANTES}

Se analizaron los partidos correspondientes a la fase final del Campeonato del Mundo masculino celebrado en 2019: Dinamarca-Francia (semifinal), NoruegaAlemania (semifinal), Francia-Alemania (partido por la medalla de bronce) y Dinamarca-Noruega (final). Este estudio se desarrolló siguiendo los principios éticos establecidos en la Declaración de Helsinki $y$, de acuerdo con lo establecido en el Informe Belmont (1978), no fue necesaria la obtención de consentimiento informado ni el examen del comité ético pertinente porque: (a) el estudio implicó la observación de personas en un entorno público (recinto deportivo); (b) las personas y los grupos observados no tenían una expectativa razonable de privacidad (los partidos fueron retransmitidos a nivel mundial); y (c) el estudio no implicó intervención por parte de los investigadores o interacción directa con los individuos estudiados.

\subsection{INSTRUMENTOS}

Para registrar los comportamientos más relevantes para la consecución de los objetivos de investigación propuestos, se construyó un instrumento de observación ad hoc (tabla 1). El instrumento de observación combinó el formato de campo con un sistema de categorías exhaustivas y mutuamente excluyentes. Esta combinación permite aprovechar los puntos fuertes de ambos instrumentos, por un lado, el sistema de categorías ofrece consistencia teórica, mientras que el formato de campo, aporta flexibilidad a la hora de recoger la diversidad de comportamientos realizados por los jugadores (Anguera \& Hernández-Mendo, 2013). Las unidades de observación comenzaron con el inicio de todos los ataques posicionales y concluyeron una vez finalizada la fase de repliegue defensivo.

Los partidos analizados fueron obtenidos directamente de internet, el programa informático Dartfish 5.5 permitió la aplicación del instrumento de observación en el registro y codificación de los partidos. Para valorar la bondad de las categorías, determinar a fiabilidad de los observadores y establecer el número mínimo de partidos necesario para generalizar con precisión, se realizó el análisis de generalizabilidad con el programa informático EduG versión 6.1. Posteriormente, se utilizó el programa informático SDIS-GSEQ 4.0 (Bakeman \& Quera, 1995) para analizar la calidad de dato y realizar el análisis secuencial, requisito previo al cálculo de coordenadas polares, que fue realizado por el programa Hoisan 1.2 (Hernández-Mendo, López, Castellano, Morales \& Pastrana, 2012). Por último, la representación gráfica se de los distintos mapas de coordenadas polares se llevó a cabo con el Snowflake 0.2.

\begin{tabular}{|c|c|}
\hline \multicolumn{2}{|c|}{ Tabla 1. Instrumento de observación } \\
\hline Criterio & Categorías \\
\hline $\begin{array}{c}\text { Equipo } \\
\text { (EQU) }\end{array}$ & Equipo al que pertenece la unidad de observación analizada: \\
\hline $\begin{array}{c}\text { Portería vacía } \\
\text { (PVA) }\end{array}$ & Se juegarca con portería vacía (SI)/ No se juega con portería vacía(NO) \\
\hline $\begin{array}{c}\text { Número } \\
\text { (REL) }\end{array}$ & Relación numérica entre ambos equipos: \\
\hline
\end{tabular}




\begin{tabular}{|c|c|}
\hline $\begin{array}{l}\text { Número de } \\
\text { jugadores con los } \\
\text { que juega cada } \\
\text { equipo (NUM) }\end{array}$ & $\begin{array}{c}\text { Portería vacía } 7 \text { vs.6 (P7x6)/ Portería vacía } 7 \text { vs.5 (P7x5)/ Portería vacía } \\
\text { (P6x5)/ Portería vacía } 6 \text { vs.6 (P6x6)/ Portería vacía (P5x6)/ Portería vacía } \\
5 \text { vs.5 (P5x5)/ Portería vacía (P5x4)/ No portería vacía } 6 \text { vs.6 (NP6x6)/ No } \\
\text { portería vacía } 6 \text { vs. } 5 \text { (NP6x5)/ No portería vacía } 5 \text { vs.6 (NP 5x6)/ No } \\
\text { portería vacía } 5 \text { vs. } 5 \text { (NP5x5)/ No portería vacía de } 6 \text { vs.4 (NP6x4). }\end{array}$ \\
\hline $\begin{aligned} \text { Marca } \\
\text { (MA }\end{aligned}$ & $\begin{array}{c}\text { El equipo analizado va: Ganando de por una diferencia no superior a } 3 \\
\text { goles (GD3)/ Ganando de por una diferencia superior a } 3 \text { goles (GM3)/ } \\
\text { Ganando de por una diferencia superior a } 6 \text { goles (GM6)/ Perdiendo por } \\
\text { una diferencia no superior a } 3 \text { goles (PD3)/ Perdiendo por una diferencia } \\
\text { superior a } 3 \text { goles (PM3)/ Perdiendo por una diferencia superior a } 3 \text { goles } \\
\text { (PM6)/ Empate (EPT) }\end{array}$ \\
\hline $\begin{array}{l}\text { Tiempo de } \\
\text { partido } \\
\text { (TIE) }\end{array}$ & $\begin{array}{c}\text { Momento del partido en el que se inicia el ataque analizado: Primeros } 20 \\
\text { minutos de la primera parte (PRI20)/ Últimos } 10 \text { minutos de la primera } \\
\text { parte (PRI30)/ Primeros } 20 \text { minutos de la segunda parte (SEG20)/ Últimos } \\
10 \text { minutos de la segunda parte hasta final partido (SEG30). }\end{array}$ \\
\hline $\begin{array}{l}\text { Resultado Ataque } \\
\text { (RES) }\end{array}$ & $\begin{array}{l}\text { Desenlace del ataque: El ataque finaliza con gol, } 7 \text { metros o exclusión de } \\
2 \text { minutos de un defensor (G72)/ El ataque finaliza en un golpe franco, } \\
\text { saque de banda, córner o interrupción de los árbitros in que el equipo } \\
\text { pierda la posesión de balón (GFC)/ Lanzamiento que no acaba en gol } \\
\text { (L6N)/ El ataque finaliza con una pérdida de balón (PER) }\end{array}$ \\
\hline $\begin{aligned} \text { Mov } \\
(\mathrm{M}\end{aligned}$ & $\begin{array}{l}\text { Número de jugadores que intercan } \\
\text { Ninguno (JG0)/ dos (JG2)/ tres }\end{array}$ \\
\hline $\begin{array}{l}\text { Secu } \\
\text { ata } \\
(\mathrm{SC}\end{array}$ & $\begin{array}{l}\text { Número de intentos, en una misma posesió } \\
\text { equipo. Se considera que comienza un nuel } \\
\text { realización de un lanzamiento o de que se inter } \\
(\mathrm{SC} 1) / \text { dos intentos (SC2)/ tres intentos (SC }\end{array}$ \\
\hline $\begin{array}{r}\text { Acción } \\
\text { finalizacic } \\
\text { ataque pos } \\
(\mathrm{ACC}\end{array}$ & $\begin{array}{c}\text { El acción con la que finaliza el ataque: Juego con pivote (PVT)/ Juego } \\
\text { hacia alguno de los extremos (EXT)/ Penetración o finta de uno de los } \\
\text { jugadores de la primera línea (J6M)/ Juego desde en las proximidades de } \\
\text { la línea de } 9 \text { metros (J9M) }\end{array}$ \\
\hline $\begin{array}{l}\text { Sistema de } \\
\text { ataque } \\
\text { (SIS) }\end{array}$ & $\begin{array}{c}\text { Sistema de ataque empleados en el ataque: Uno de los extremos } \\
\text { abandona su posición y para ocupar la posición del pivote (DEX)/ Uno de } \\
\text { los jugadores de la primera línea abandona su posición para ocupar la } \\
\text { posición de pivote (D1L)/ Ninguno de los jugadores abandona su posición } \\
\text { para ocupar la posición de pivote (NOD)/ se inicia el ataque con dos } \\
\text { pivotes específicos (2PV) }\end{array}$ \\
\hline $\begin{array}{l}\text { Zona Finalización } \\
\text { (ZON) }\end{array}$ & $\begin{array}{l}\text { Al dividir el campo en tres zo } \\
\text { ataque: Izquierda (IZQ)/ Z }\end{array}$ \\
\hline $\begin{array}{l}\text { Sistema } \\
\text { Defensivo } \\
\text { (DEF) }\end{array}$ & $\begin{array}{c}\text { Sistema defensivo empleado por el equipo defensivo: 6:0 (SEI)/ Sistema } \\
\text { defensivo con defensor avanzado (AVN)/ 5:0 (CIN)/ Sistema defensivo } \\
4: 2,3: 3 \text { o individual (ABI)/ Sistema defensivo mixto (MIX) }\end{array}$ \\
\hline $\begin{array}{l}\text { Cruces/Permutas } \\
(\text { XYP) }\end{array}$ & $\begin{array}{l}\text { Número de cruces y/o permutas realizadas en el ataque: Cero (CP0)/ } \\
\text { Uno (CP1)/ Dos (CP2)/ Tres (CP3)/ Cuatro (CP4)/ Cinco (CP5) }\end{array}$ \\
\hline $\begin{array}{l}\text { Contraataque } \\
\text { (CTQ) }\end{array}$ & $\begin{array}{l}\text { Realización de contraataque por parte del equipo que estaba en fase } \\
\text { defensiva: Hay contraataque (SIHAY)/ No hay contraataque (NOHAY) }\end{array}$ \\
\hline $\begin{array}{l}\text { Inicio del } \\
\text { contraataque } \\
(\mathrm{INI})\end{array}$ & $\begin{array}{l}\text { Situación a partir de la que se inicia el contraataque: } \\
\text { Después de un lanzamiento que no acaba en gol (PAR)/ Después de una } \\
\text { recuperación balón (RCU)/ Después de encajar gol (GOL) }\end{array}$ \\
\hline $\begin{array}{l}\text { Tipo } \\
\text { (TIP) }\end{array}$ & $\begin{array}{l}\text { Tipo de contraataque realizado: Primera oleada (1OL)/ Segunda oleada } \\
\text { (2OL)/ Saque de centro (SCN)/ Lanzamiento a portería vacía (CPP) }\end{array}$ \\
\hline $\begin{array}{l}\text { Resultado del } \\
\text { contraataque } \\
\quad(\text { RCQ) }\end{array}$ & $\begin{array}{c}\text { Desenlace del contraataque: Gol, señalización de } 7 \text { metros o exclusión de } \\
2 \text { minutos a un jugador en fase de repliegue (CEX)/ Lanzamiento que no } \\
\text { acaba en gol (MIS)/ Pérdida de balón (CPR)/ El contraataque se para y } \\
\text { comienza la fase de ataque posicional (STP) }\end{array}$ \\
\hline Cambios equipo & Número de cambios que realiza el equipo en fase de repliegue defensivo: \\
\hline
\end{tabular}




\begin{tabular}{|c|c|}
\hline $\begin{array}{c}\text { repliegue } \\
(\mathrm{CAM})\end{array}$ & Un cambio (UNO)/ Dos cambios (DOS)/ Tres cambios (TRE)/ Ningún \\
cambio (CRO)
\end{tabular}

\subsection{PROCEDIMIENTO}

\subsubsection{CALIDAD DEL DATO}

Un observador principal (entrenador nacional de balonmano, con experiencia en metodología observacional y autor del instrumento de observación diseñado para este estudio) llevó a cabo los registros de cada uno de los partidos. Con el objetivo de analizar la calidad de los datos, otro observador, entrenador nacional de balonmano y con dominio en la utilización del instrumento de registro, realizó un proceso de entrenamiento que consistió en dos fases: explicación de los criterios y categorías del instrumento de observación y registro de partidos de balonmano que no fueron incluidos en la muestra. El proceso de entrenamiento concluyó cuando, se obtuvo un nivel de concordancia intraobservador (un mismo partido registrado por el mismo observador en dos momentos distintos) e interobservadores (un mismo partido registrado por el observador principal y por el observador entrenado) superior a 0,80 en el estadístico Kappa de Cohen, para todos los criterios del instrumento de observación. Para el cálculo de concordancia intraobservador, así como para el cálculo de la concordancia interobservadores, se utilizó uno de los partidos correspondientes a la segunda fase del campeonato, obteniéndose en ambos casos un índice Kappa de Cohen superior a 0,96 en todos los criterios y en ambas pruebas de fiabilidad (intra e inter). De acuerdo el baremo de Landis \& Koch (1977), el nivel de acuerdo mostrado puede considerarse "casi perfecto".

\subsubsection{ANÁLISIS DE GENERALIZABILIDAD}

Para valorar la bondad de las categorías, determinar a fiabilidad de los observadores y establecer el número mínimo de partidos necesario para generalizar con precisión, se realizó el análisis de generalizabilidad (Cronbach, Gleser, Nanda \& Rajaratnam, 1972). Se planteó un diseño de dos facetas Partidos / Categoría (P/C), para determinar la homogeneidad de las categorías. Se obtuvieron unos coeficientes de generalización próximos a cero: 0,20 el relativo y 0,05 el absoluto; estos valores indican que las categorías son adecuadas y cumplen con el requisito de ser exhaustivas y mutuamente excluyentes. Por otra parte, el diseño de dos facetas Categoría / Partidos (C/P) obtuvo unos coeficientes de generalización, tanto absolutos como relativos, de 0.93, estos valores indican que con la observación de 4 partidos se obtiene un alto grado de generalización. Asimismo, el análisis del modelo lineal generalizado mostró que la mayor parte de la varianza se explicaba por el componente de categorías $(78,2 \%)$ y su interacción con el componente de partidos $(21,8 \%)$, lo que demuestra la confiabilidad interpretativa de los datos registrados en las diferentes categorías en los distintos partidos. 


\subsubsection{ANÁLISIS DE COORDENADAS POLARES}

El análisis de coordenadas polares permite conocer los patrones de conducta que emergen en los deportes de equipo (Flores-Rodríguez \& Anguera, 2018). Como paso previo al análisis de coordenadas polares, se requiere el cálculo de los residuos ajustados mediante la aplicación del análisis secuencial. El objetivo del análisis secuencial es identificar las relaciones significativas, tanto a nivel prospectivo como retrospectivo, entre distintos comportamientos que son considerados de interés por parte de los investigadores. Ambas perspectivas tienen que ser consideradas para poder representar gráficamente las relaciones entre las categorías estudiadas (Gorospe \& Anguera, 2000). Entre las categorías objeto de análisis, se denomina conducta focal, a la que es considerada como generadora "hacia adelante" (perspectiva prospectiva) y "hacia atrás" (perspectiva retrospectiva) de las asociaciones con las otras conductas estudiadas, que asumen el rol de conductas condicionadas (Tarragó et al., 2017). Por medio de una potente técnica de reducción de datos, el cálculo del estadístico $Z_{\text {sum }}=\frac{\sum Z}{\sqrt{n}}$ (Sackett, 1980), se conoce tanto la fuerza de la asociación, indicada por la longitud del vector, como la naturaleza de la misma (activación o inhibición), indicada por el cuadrante en el que queda ubicada la asociación. Cuando la asociación es representada en el cuadrante I, indica una relación de activación mutua entre la conducta focal y la condicionada; si la representación queda ubicada en el cuadrante IV indica que la conducta focal activa a la condicionada, mientras que esta última inhibe a la focal; si la asociación es representada en el cuadrante III, existe una relación de inhibición mutua entre las conductas estudiadas; y, por último, cuando la representación gráfica se realiza en el cuadrante II, implica que la conducta focal inhibe a la condicionada y esta última activa a la focal (Anguera et al., 2011).

\section{RESULTADOS}

En un primer apartado se expondrá un análisis descriptivo sobre el porcentaje de ataques posicionales donde se ha utilizado la nueva norma, el porcentaje de acierto obtenido y porcentaje de contraataques con éxito (realizados por el equipo que defendía). Posteriormente en un segundo apartado, se estudiaran los patrones de conducta que emergen en una situación de 6 vs. 6 , jugada con portería vacía (cuando el equipo analizado está en inferioridad) y jugada sin que se haya realizado la sustitución del portero por un jugador de campo (ambos equipos en igualdad).

\subsection{ANÁLISIS DESCRIPTIVO}

Un total de 445 unidades de observación han sido analizadas en este estudio. La tabla 2 recoge el análisis descriptivo referente a la utilización y éxito (finalización en gol, señalización de 7 metros o exclusión de un jugador rival) del ataque y del contraataque, obtenidos cuando se utiliza y cuando no se utiliza el recurso estratégico de jugar con la portería vacía. Los datos se presentan diferenciando la relación numérica entre ambos equipos (igualdad, 
superioridad e inferioridad) y considerando el total de las acciones en su conjunto.

Podemos observar como las acciones totales con portería vacía suponen el $17,1 \%$ del total de acciones analizadas, obteniendo un menor porcentaje de éxito en ataque y un mayor porcentaje de éxito en los contraataques recibidos, respecto a la no utilización de la portería vacía. Asimismo, destaca la utilización de la nueva norma en el $100 \%$ de las acciones se juegan en inferioridad numérica.

\begin{tabular}{|c|c|c|c|c|c|c|c|}
\hline \multirow[b]{2}{*}{ R.N } & \multirow[b]{2}{*}{ N. A } & \multicolumn{3}{|c|}{ No Portería Vacía } & \multicolumn{3}{|c|}{ Portería Vacía } \\
\hline & & Utilización & E.A & E.C. & Utilización & E.A & E.C \\
\hline Igualdad & 348 & $\begin{array}{c}(332) \\
95,4 \%\end{array}$ & $\begin{array}{c}(130) \\
39,2 \%\end{array}$ & $\begin{array}{l}(43) \\
13 \%\end{array}$ & $\begin{array}{l}(16) \\
4,6 \%\end{array}$ & $\begin{array}{c}(5) \\
31,3 \%\end{array}$ & $\begin{array}{c}(3) \\
18,8 \%\end{array}$ \\
\hline Superioridad & 40 & $\begin{array}{c}(37) \\
92,5 \%\end{array}$ & $\begin{array}{c}(21) \\
56,8 \%\end{array}$ & $\begin{array}{l}(0) \\
0 \%\end{array}$ & $\begin{array}{c}(3) \\
7,5 \%\end{array}$ & $\begin{array}{c}(2) \\
66,7 \%\end{array}$ & $\begin{array}{l}(0) \\
0 \%\end{array}$ \\
\hline Inferioridad & 57 & $\begin{array}{l}(0) \\
0 \% \\
\end{array}$ & $\begin{array}{l}(0) \\
0 \% \\
\end{array}$ & $\begin{array}{l}(0) \\
0 \% \\
\end{array}$ & $\begin{array}{c}(57) \\
100 \% \\
\end{array}$ & $\begin{array}{c}(20) \\
35,1 \% \\
\end{array}$ & $\begin{array}{c}(13) \\
22,8 \% \\
\end{array}$ \\
\hline TOTAL & 445 & $\begin{array}{c}(369) \\
82,9 \% \\
\end{array}$ & $\begin{array}{r}(151) \\
40,9 \% \\
\end{array}$ & $\begin{array}{c}(43) \\
11,7 \% \\
\end{array}$ & $\begin{array}{c}(76) \\
17,1 \% \\
\end{array}$ & $\begin{array}{c}(27) \\
35,5 \% \\
\end{array}$ & $\begin{array}{c}(16) \\
21,1 \% \\
\end{array}$ \\
\hline
\end{tabular}

Al analizar específicamente la situación de 6 vs. 6 (tabla 3), se advierte que el porcentaje de éxito del ataque es menor cuando se utiliza el recurso de portería vacía que cuando no se utiliza. También se aprecia que al $23,6 \%$ de los ataques realizados con portería vacía le siguen contraataques exitosos, situación que se reduce al $13 \%$ cuando los ataques no se juegan con portería vacía.

\begin{tabular}{c|cc|c|cc}
\hline \multicolumn{3}{c}{ Tabla 3. 6 vs. 6 } \\
\hline \multicolumn{3}{c}{$\begin{array}{c}\text { No Portería Vacía } \\
\text { (NP6x6) }\end{array}$} & \multicolumn{3}{c}{$\begin{array}{c}\text { Portería Vacía } \\
\text { (P6x6) }\end{array}$} \\
\hline N.A & E.A. & E.C. & N.A & E.A & E.C \\
332 & $(130)$ & $(43)$ & $(55)$ & $(18)$ & $(13)$ \\
& $39,2 \%$ & $13 \%$ & & $32,7 \%$ & $23,6 \%$ \\
\hline
\end{tabular}

\subsection{ESTUDIO DE LOS PATRONES DE CONDUCTA}

A continuación, se estudiaron los patrones de conducta que emergen en una situación de 6 vs. 6 jugada con portería vacía (cuando el equipo que ataca está en inferioridad numérica y sustituye al portero por un jugador de campo para compensar la ausencia del jugador excluido) comparándolos con los que emergen en la misma situación, de 6 vs. 6 , sin que se haya realizado la sustitución del portero por un jugador de campo.

Por medio del análisis de coordenadas polares, se conocen y representan gráficamente las asociaciones significativas, aquellas con una longitud $>1,96$ 
$(p<0,05)$, entre la conducta focal y las condicionas. Los mapas de coordenadas polares están distribuidos en 4 tablas. Para facilitar la comparación entre los diferentes patrones de conducta que emergen en las situaciones analizadas, en cada tabla se presentarán dos mapas, el de la izquierda tendrá como conducta focal NP6x6 (ataques que no se desarrollan con la portería vacía), mientras que el de la derecha tendrán como conducta focal P6x6 (ataques que se juegan con la portería vacía).

En la tabla 4, actúan como conductas condicionadas, las categorías pertenecientes a los criterios MOV (número de jugadores que intercambian sus posiciones) y SCN (número de intentos). Cuando las selecciones juegan sin sustituir al portero (NP6x6) muestran una asociación de activación recíproca (cuadrante I) con la finalización de los ataques en su cuarto intento (SC4) y con ataques donde los jugadores no intercambian sus posiciones (JG0), pero en este caso la activación no es mutua (cuadrante IV). Por otro lado, en el cuadrante III, se muestra una asociación de inhibición mutua con: ataques donde 4 jugadores intercambian su posición (JG4) y con ataques que finalizan en su tercer intento (SC3). Asimismo, la conducta focal NP6x6 inhibe la

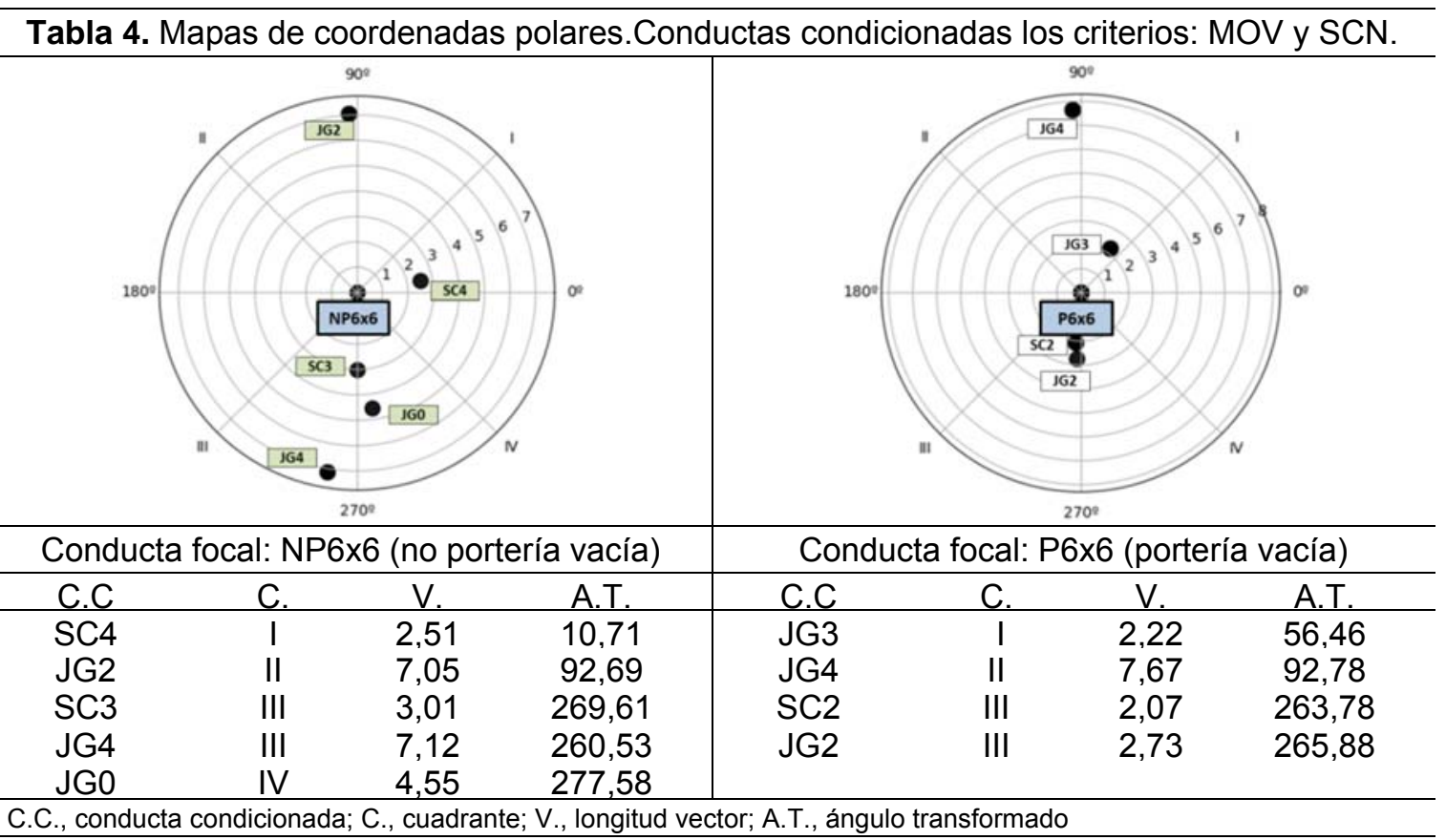

aparición de ataques donde 2 jugadores intercambian sus posiciones (JG2), pero en este caso la inhibición no es mutua (cuadrante II). En el mapa de la derecha, cuando el juego se desarrolla con la portería vacía (P6x6), se observa una mutua activación con la realización de ataques donde 3 jugadores intercambian sus posiciones (JG3). Por contra, podemos observar una asociación de inhibición mutua (cuadrante III) con ataques donde 2 jugadores intercambian sus posiciones (JG2) y con ataques que finalizan en su segundo intento (SC2). Asimismo, la presencia de la conducta focal inhibe la aparición de ataques donde 4 jugadores intercambian sus posiciones (JG4), aunque en este caso la inhibición no es mutua (cuadrante II). 
En la tabla 5 actúan como conductas condicionadas las conductas pertenecientes a los criterios SIS (sistemas de ataque) y XYP (cruces y permutas). En el mapa de la izquierda, cuando la conducta focal es NP6x6 (no se sustituye al portero), se muestra una asociación de activación mutua (cuadrante I) con ataques donde ningún jugador (ni extremos ni primeras líneas) abandona su posición para ocupar el puesto de pivote (NOD) y con ataques donde se realiza un cruce o permuta (CP1). Por otro lado, se aprecia una relación de inhibición mutua (cuadrante III) con la utilización de un sistema de ataque que cuenta inicialmente con 2 jugadores en la posición de pivote (2PV) y con ataques donde se realizan 4 cruces y/o permutas (CP4). También podemos ver en el cuadrante II, como la conducta focal inhibe la aparición de ataques donde uno de los extremos abandona su posición para ocupar la posición de pivote (DEX) y con ataques donde se realizan 2 cruces o permutas (CP2), aunque en este caso la inhibición no es mutua. En el mapa de la derecha, cuando el juego se desarrolla con portería vacía (P6x6), se observa una mutua activación (cuadrante I) con ataques donde se realizan 4 cruces o permutas (CP4). También se aprecia como la conducta focal activa los ataques donde uno de los extremos circula a la posición de pivote (DEX) y con ataques donde se realizan 1 cruce o permuta (CP1), pero en este caso la activación no es mutua (cuadrante IV). Por el contrario la conducta focal inhibe la aparición de ataques que se ninguno de los extremos o primeras líneas circula a la posición de pivote (NOD) y con la realización de 3 cruces y/o permutas (CP3).

Tabla 5. Mapas de coordenadas polares. Conductas condicionadas los criterios: SIS y XYP

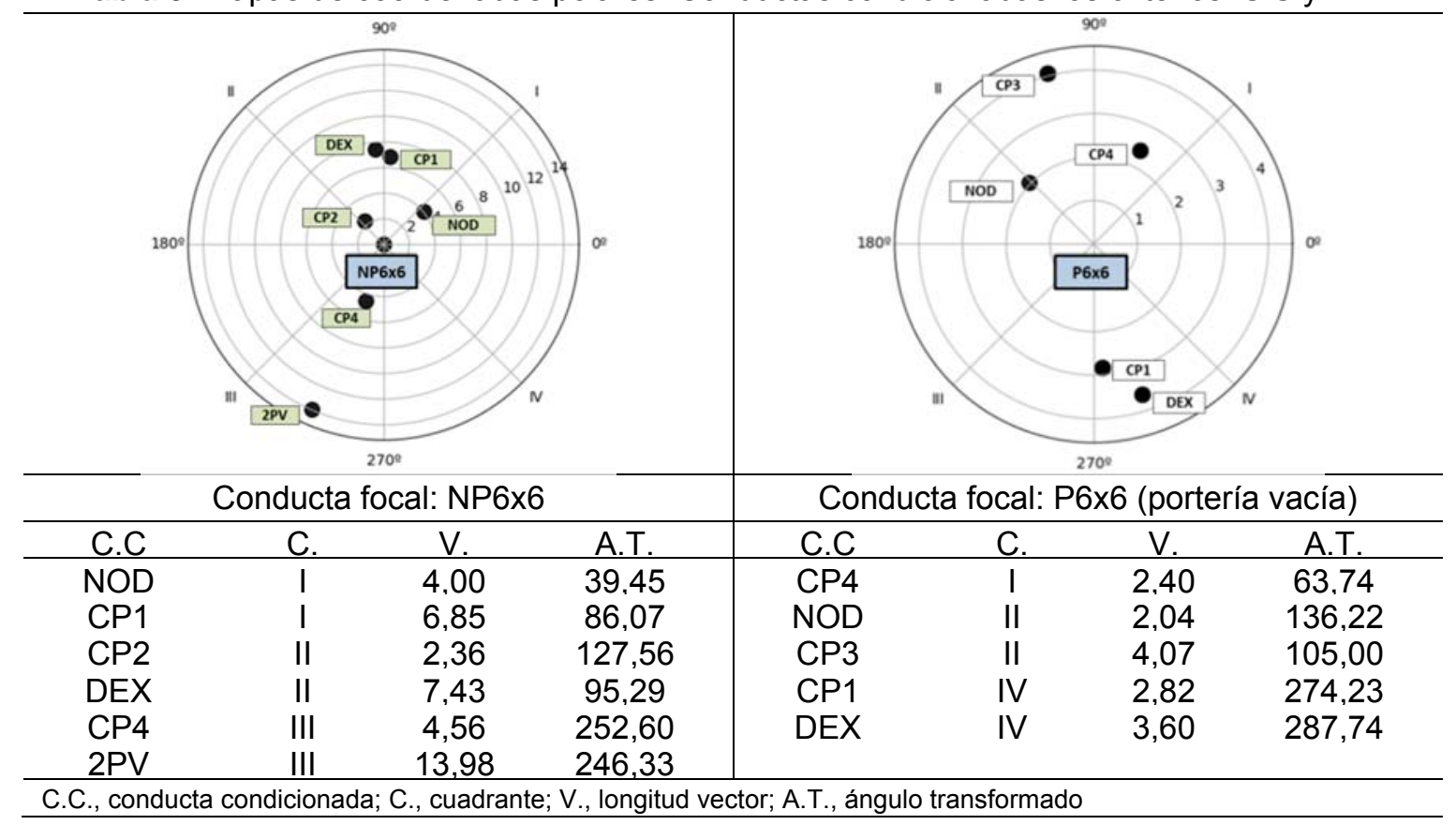

En la tabla 6 actúan como conductas condicionadas las categorías pertenecientes a los criterios ZON (zona del campo donde finaliza el ataque posicional), ACC (acción con la que finaliza el ataque) y RES (resultado del ataque posicional). Cuando la conducta focal es NP6x6 (no se sustituye al portero) se muestra una asociación de activación mutua (cuadrante I) con la ataques que finalizan las proximidades de la línea de 9 metros (J9M), en la zona central (CEN) y con ataques que finalizan con una interrupción (GFC). 
Asimismo, la conducta focal también activa la finalización de ataques por medio de fintas o penetraciones, realizadas por los jugadores que forman la primera línea (J6M) y con la finalización de los ataques en gol, señalización de 7 metros o exclusión de un defensor (G72), pero en estos casos la activación no es mutua (cuadrante IV). Por otro lado, la conducta focal presenta una relación de inhibición mutua (cuadrante III) con ataques que finalicen por medio de acciones de pivote (PVT) o por medio de acciones de extremo (EXT). La conducta focal también inhibe la aparición de ataques que finalicen con pérdida de balón (PER) y con lanzamientos fallados (L6N), aunque estas asociaciones de inhibición no son recíprocas. En el mapa de la derecha, cuando las acciones se realizan con la portería vacía (P6×6), se observa una mutua activación mutua (cuadrante I) con la finalización de los ataques en gol, señalización de 7 metros o exclusión de un defensor rival (G72) y una relación de inhibición recíproca (cuadrante III) con la finalización de los ataques con una interrupción (GFC).

Tabla 6. Mapas de coordenadas polares. Conductas condicionadas criterios: ZON, ACC y RES

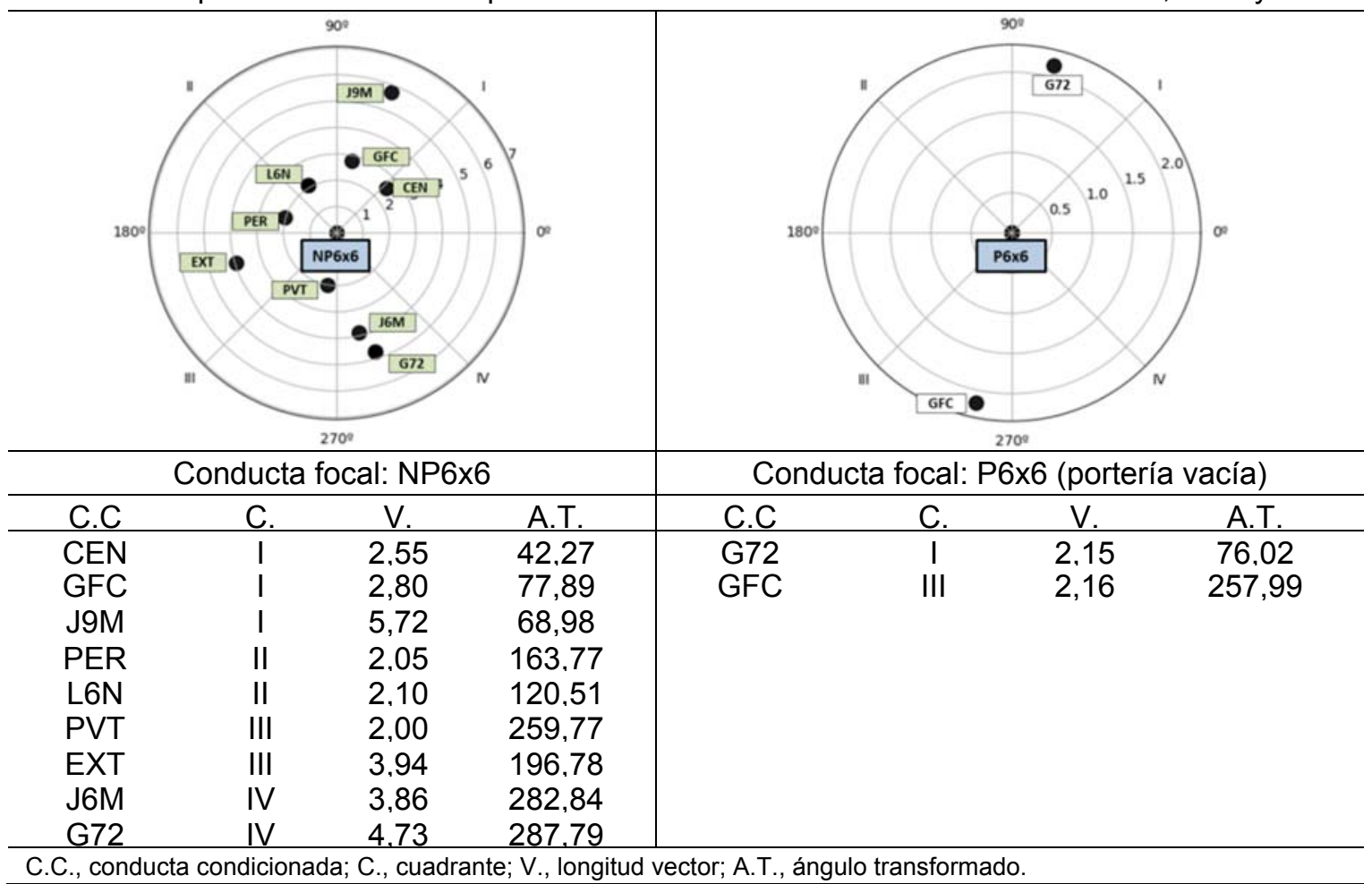

Por último, en la tabla 7 actuaron como conductas condicionadas, las categorías pertenecientes a los criterios relacionados con el contraataque (realizado por el equipo que defendía) subsiguiente al ataque posicional: INI (modo de inicio del contraataque), TIP (tipo de contraataque realizado) y RCQ (resultado del contraataque). En el mapa de la izquierda, cuando la conducta focal es NP6x6 (no se sustituye al portero), se muestra una asociación de activación mutua (cuadrante I) con la realización de saques de centro rápidos (SCN) después de recibir un gol (GOL), finalización de contraataques con una pérdida de balón (CPR) y con contraataques que se paran y para dar comienzo a un ataques posicional (STP). Por otro lado, se aprecia una relación de inhibición mutua (cuadrante III) con contraataques que finalizan en gol, 7 
metros o exclusión de un defensor (CEX) y con la realización de un lanzamiento a portería vacía (CPP). También podemos ver en el cuadrante II, como la conducta focal inhibe la aparición de contraataques que se inician después de una recuperación de balón (RCU) y con la realización de contraataques en segunda oleada (2OL), aunque en este caso la inhibición no es mutua. En el mapa de la derecha, cuando la conducta focal es P6x6 (se sustituye al portero), se muestra una asociación de activación mutua (cuadrante I) con las: realización de lanzamientos a portería vacía (CPP) y con la finalización de los contraataques en gol, señalización de 7 metros 0 exclusión de un jugador rival (CEX). Asimismo, la conducta focal activa la finalización de contraataques que finalizan en pérdida de balón, pero en este caso la activación no es mutua (cuadrante IV). Por contra, se aprecia una relación de inhibición mutua (cuadrante III) con contraataques que se paran, para dar paso a la realización de un ataque posicional (STP).

Tabla 7. Mapas de coordenadas polares. Conductas condicionadas criterios: INI, TIP Y RCQ

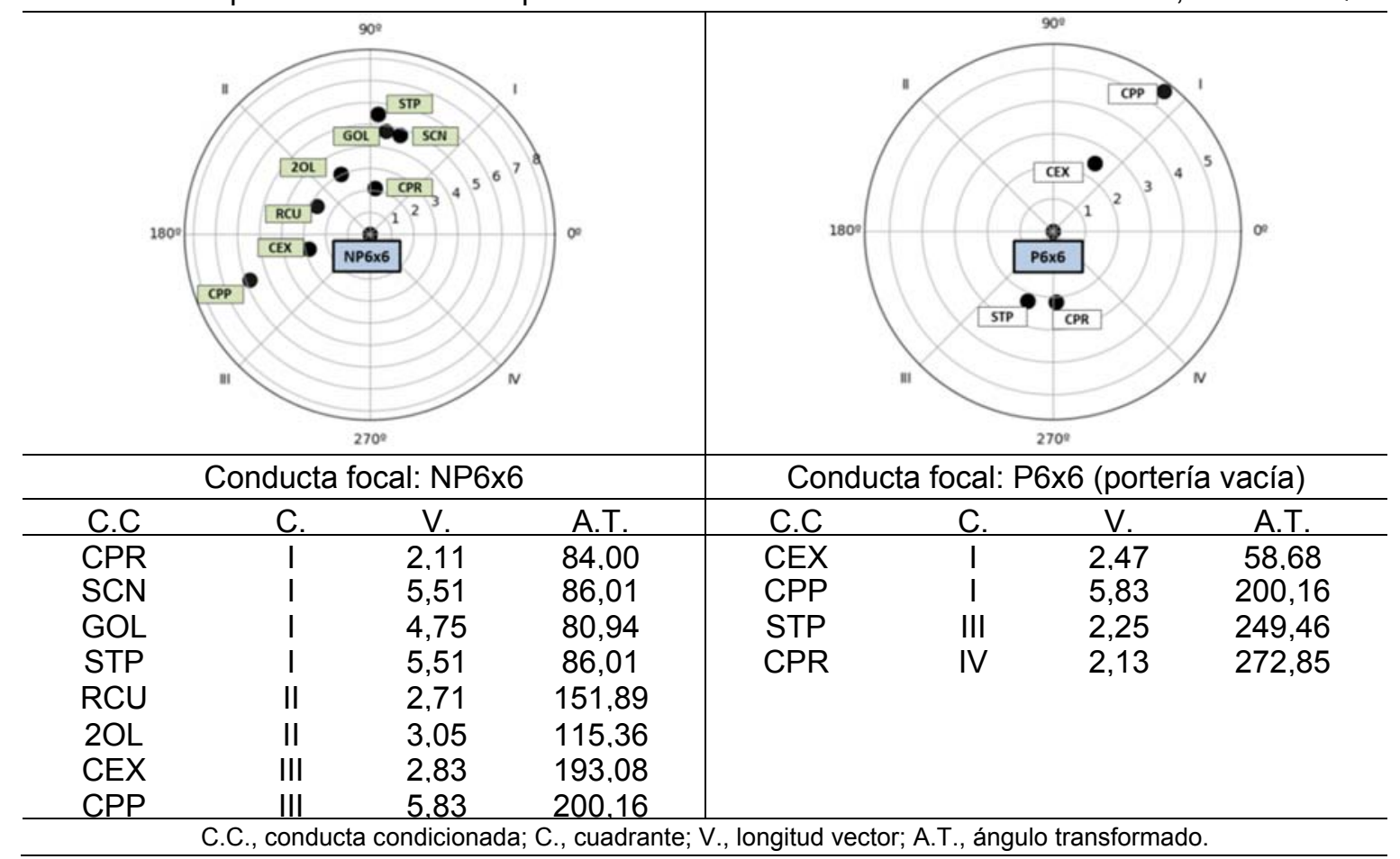

\section{DISCUSIÓN}

La presente investigación pretende conocer el impacto ejercido por la nueva regla, que permite sustituir al portero por un jugador de campo extra asumiendo el riesgo de jugar con la portería vacía, en los partidos correspondientes a la fase final (semifinales, final y partido por el tercer puesto) del Campeonato del Mundo de masculino celebrado en Dinamarca y Alemania en 2019. Debido a lo reciente de la implantación de la norma, no son muchos los trabajos con los que poder comparar los resultados obtenidos en nuestra investigación.

El análisis descriptivo nos muestra que la nueva norma es utilizada en el $17,1 \%$ de los ataques, porcentaje ligeramente inferior al $19,98 \%$ utilizado en el Campeonato del Mundo masculino celebrado en 2017 (Marczinka \& Gál, 2018). 
Cuando ambos equipos están en igualdad numérica, la sustitución del portero por un jugador de campo extra para obtener una ventaja numérica respecto a la defensa, sólo es utilizada en el $4,6 \%$ de los ataques. No parece un recurso ventajoso, pues el ataque posicional obtiene un porcentaje de éxito del $31,3 \%$ cuando se juega con portería vacía, porcentaje inferior al 39,2\% obtenido en los ataques donde la nueva norma no es empleada. Asimismo, jugar con la portería vacía penaliza la fase de repliegue, pues en el $18,8 \%$ de los ataques, el contraataque subsiguiente obtiene éxito, siendo un porcentaje superior al $13 \%$ obtenido cuando no se juega con portería vacía.

En todas las situaciones de inferioridad numérica analizadas se utiliza el recurso de la portería vacía. Antes de la entrada en vigor de la nueva norma, (Beiztegui-Casado et al., 2019) encontraron que la sustitución del portero por un jugador de campo se producía en el $16,6 \%$ de los ataques en inferioridad, por lo que podemos comprobar que la nueva norma ha disparado la utilización de este recurso. De acuerdo con Marczinka \& Gál (2018), parece ser que los equipos principalmente utilizan el recurso de portería vacía, no con el objetivo de jugar los ataques en superioridad numérica, sino con el objetivo de compensar la ausencia del jugador excluido.

Por otro lado, el análisis de coordenadas polares ha demostrado que no es lo mismo jugar una situación de 6 vs. 6 (seis atacantes contra seis defensores) con la portería vacía (en inferioridad numérica) que jugarla con portero (en igualdad numérica). Los patrones de conducta que emergen en el ataque posicional difieren considerablemente según esté o no la portería vacía. La condición de portería vacía activó ataques con una mayor movilidad: más jugadores intercambiaron sus posiciones, se realizaron más cruces y permutas, y se activó la aparición de ataque donde uno de los extremos circulaba a la posición de pivote. Comportamientos muy diferentes a los desarrollados cuando el 6 vs. 6 es jugado sin que esté la portería vacía, pues en esta situación los ataques son más directos: se realizan pocos cruces y/o permutas, no son activados comportamientos relacionados con la transformación del sistema de ataque 3:3 a otros sistemas con dos pivote y se muestra una tendencia a finalizar los ataques mediante acciones de la primera línea, ya sea con acciones desde los 9 metros o 6 metros, en la zona central del ataque. Estos comportamientos favorecen la realización de golpes francos por parte de la defensa, a la misma vez que inhiben las finalizaciones mediante acciones de pivote o extremo, comportamientos que no son inhibidos cuando se juega con la portería vacía.

Respecto a la fase de repliegue, la situación de 6 vs. 6 con portería vacía penaliza la fase de repliegue respecto a la situación 6 vs. 6 sin portería vacía, pues en el $23,6 \%$ de los ataques realizados con portería vacía, el equipo que defendía tiene éxito en el contraataque, mientras que en la situación de 6 vs. 6 con portero el porcentaje es del $13 \%$. El análisis de coordenadas polares refleja un desarrollo de los contraataques muy distintos en las dos situaciones estudiadas. Cuando la portería está vacía, se activa la finalización con éxito de los mismos y la realización de lanzamientos lejanos a portería que está sin portero. Por otra parte, cuando se juega el 6 vs. 6 sin portería vacía, se activan los contraataques iniciados después de encajar un gol, mediante la realización 
de un saque de centro rápido. Respecto a los comportamientos relacionados con la finalización, se activan la aparición de contraataques que se paran para que comience la fase de ataque posicional y se inhiben los contraataques que finalizan con éxito. Estos resultados indican que realizar la fase de repliegue en inferioridad numérica, junto con la realización de, al menos, una sustitución de un jugador de campo por el portero, provoca una situación muy favorable para el equipo que contraataca, lo que supone diferencias importantes respecto a los resultados encontrados por (Beiztegui-Casado et al., 2019). De igual modo, llama la atención que en situaciones de 6 vs. 6 con portería vacía los contraataques iniciados después de gol, mediante un rápido saque de centro, no son activados; la razón podría ser que los equipos prefieren realizar un ataque posicional en superioridad numérica, antes que asumir los riesgos de este tipo de contraataque.

Los entrenadores necesitan tiempo para responder tácticamente a las posibilidades ofrecidas por la implantación de nuevas reglas. Los equipos, con el objetivo de adaptarse a los nuevos contextos generados (nueva dinámica de juego), van afianzando determinados comportamientos (estrategias, sistemas de juego, tácticas) y descartando otros, por lo que, la obtención de conocimiento científico que ayude a comprender hacia donde se dirige el juego, puede servir de ayuda a los entrenadores, ya sean de etapas de formación o rendimiento, en el diseño de sus programas de entrenamiento, así como a los investigadores en su labor científica. Por último, creemos que es necesaria la realización de futuros estudios que sigan profundizando en el impacto de esta norma en el desarrollo del juego, poniendo el foco de estudio en otras fases del juego, como la defensa por ejemplo, así como en las fases del juego analizadas en este estudio para: comprobar si las tendencias encontradas en este estudio se confirman o cambian en futuros campeonatos, conocer las tendencias desarrolladas en el ámbito del balonmano femenino, conocer las tendencias específicos que presentan determinados equipos o selecciones, así como conocer la influencia de esta norma en jugadores jóvenes.

\section{CONCLUSIONES}

Las conclusiones más importantes, en relación a los objetivos planteados, que se desprenden tras el análisis de los partidos correspondientes a la fase final del Campeonato del Mundo masculino 2019, son las siguientes:

-La sustitución del portero por un jugador de campo extra es utilizada en todos los ataques que se juegan en inferioridad numérica, para compensar la ausencia del jugador excluido.

-En situaciones de igualdad, el porcentaje de acierto de los ataques realizados con portería vacía es inferior al que tienen los ataques donde el portero no es sustituido por un jugador de campo.

-En situaciones de igualdad numérica, jugar con la portería vacía penaliza la fase de repliegue defensivo, respecto a los ataques donde no es empleado este recurso. 
-En una situación de 6 vs. 6 , los ataques que se juegan con la portería vacía (inferioridad numérica) presentan un mayor intercambio de posiciones entre los jugadores, realización de cruces y/o permutas respecto a los ataques jugados con portero (igualdad numérica), que son más directos, donde menos jugadores intercambian sus posiciones y se realizan menos cruces y/o permutas.

-En una situación de ataque 6 vs. 6 jugada con la portería vacía (inferioridad numérica), el hándicap del jugador excluido, junto a la realización de, al menos, una sustitución para que entre el portero, favorece el éxito de los contraataques.

-En una situación de 6 vs. 6 jugados sin portería vacía finalizan con acciones realizadas por los jugadores que ocupan la primera línea en la zona central del ataque, tendencias que no aparecen cuando el 6 vs. 6 se juega con portería vacía.

\section{APLICACIONES SUGERIDAS AL ENTRENAMIENTO}

Aunque las conclusiones extraídas se limitan a la competición analizada, sugerimos algunas propuestas relacionadas con las fases de ataque y repliegue. Éstas van dirigidas a situaciones donde se sustituye al portero por un jugador extra de campo para jugar una situación de 6 vs. 6:

-En ataque: elaborar el juego de manera similar a cuando se juega sin sustituir al portero (reduciendo el número de cruces y permutas sin intención de atacar a portería); no buscar exclusivamente finalizaciones desde la línea de 6 metros.

-En repliegue: reducir el número de cambios ataque-defensa; favorecer la variabilidad (diferentes espacios e inicios) en las tareas propuestas; fomentar comportamientos orientados a retrasar o interrumpir el contraataque en inferioridad numérica defensiva; anticipar la sustitución del portero.

\section{REFERENCIAS BIBLIOGRÁFICAS}

Anguera, M. T., Blanco-Villaseñor, A., Hernández-Mendo, A., \& Losada, J. L. (2011). Diseños observacionales: Ajuste y aplicación en psicología del deporte. Cuadernos de Psicología del deporte, 11(2), 63-76.

Anguera, M. T., \& Hernández-Mendo, A. (2013). La metodología observacional en el ámbito del deporte. E-Balonmano.com: Revista de Ciencias del Deporte, 9(3), 135-160.

Anton, J. L. (2010). Uso del "porero-jugador" en inferioridad numérica atacante: ¿nueva aportación táctico-estratégica?. E-Balonmano.com: Revista de Ciencias del Deporte, 6(1), 3-27.

Bakeman, R., \& Quera, V. (1995). Analyzing interaction: Sequential analysis with SDIS and GSEQ. Cambridge: Cambridge University Press.

Beiztegui-Casado, C., Oliver-Coronado, J., \& Sosa-González, P. I. (2019). Portero-Jugador En Situaciones De Inferioridad Numérica Ofensiva En 
Balonmano: ¿ Penalización O Ventaja? Rev.int.med.cienc.act.fís.deporte, 19(74), 293-307.

Cronbach, L. J., Gleser, G. C., Nanda, H., \& Rajaratnam, N. (1972). The dependability of behavioral measurements: Theory of generalizability for scores and profiles. New York, NY: Wiley.

Flores-Rodríguez, J., \& Anguera, M. T. (2018). Patrón de juego en balonmano según el jugador que ocupa la posición de central. Apunts Educación Física y Deportes, 134(4), 110-123. https://doi.org/DOI: http://dx.doi.org/10.5672/apunts.2014-0983.es.(2018/4).134.08

Gorospe, G., \& Anguera, M. T. (2000). Modificación de la técnica clásica de coordenadas polares mediante un desarrollo distinto de la retrospectividad: Aplicación al tenis. Psicothema, 12(SUPPL. 2), 279-282.

Hernández-Mendo, A., López, J. A., Castellano, J., Morales, V., \& Pastrana, J. L. (2012). HOISAN 1.2: Programa informático para uso en Metodología Observacional. Cuadernos de Psicología del Deporte, 12(1), 55-78. https://doi.org/10.4321/S1578-84232012000100006

Landis, J. R., \& Koch, G. G. (1977). The Measurement of Observer Agreement for Categorical Data. Biometrics, 33(1), 159-174.

Marczinka, Z., \& Gál, A. (2018). The Chain Reaction Between the Media and Sport. The Impact of Rule Changes in Handball. Physical Culture and Sport. Studies and Research, 80(1), 39-47. https://doi.org/10.2478/pcssr2018-0024

National Commission for the Protection of Human Subjects of Biomedical and Behavioral Research. (1978). The Belmont Report: Ethical Principles and Guidelines for the Protection of Human Subjects of Research. Recuperado de https://videocast.nih.gov/pdf/ohrp_belmont_report.pdf/

Sackett, G. P. (1980). Lag Sequential Analysis as a data Reduction Technique in social interaction research. En D. B Sawin, R. C. Hawkins, L. O. Walker \& J. H. Penticuff (Eds.), Exceptional Infant Psychosocial Risks in InfantEnvironment Transactions (pp. 300-340). New York: Brunner/Mazel.

Tarragó, R., Iglesias, X., Lapresa, D., Anguera, M. T., Ruiz-Sanchis, L., \& Arana, J. (2017). Análisis de las relaciones diacrónicas en los comportamientos de éxito y fracaso de campeones del mundo de esgrima utilizando tres técnicas complementarias. Anales de Psicología, 33(3), 471485. https://doi.org/http://dx.doi.org/10.6018/analesps.33.3.271041

Número de citas totales / Total references: 14 (100\%) Número de citas propias de la revista / Journal's own references: 1 (7,14\%)

Rev.int.med.cienc.act.fís.deporte - vol. 21 - número 83 - ISSN: 1577-0354 